\title{
BrainCheck Cognitive Assessment
}

National Cancer Institute

\section{Source}

National Cancer Institute. BrainCheck Cognitive Assessment. NCI Thesaurus. Code C154673.

A proprietary set of cognitive assessments designed to aid in diagnosing mild cognitive impairment, dementia, traumatic brain injuries, and sports-related head injuries. The tests are designed to be taken through an app on a mobile device. 